\title{
Das schwache Rationalitätsprinzip als Grundlage einer verstehenden Sozialwissenschaft ${ }^{*}$
}

\author{
GEBHARD KIRCHGÄSSNER $^{* *}$
}

Das schwache Rationalitätsprinzip ist eine heuristische Regel, wie man in den Sozialwissenschaften sinnvollerweise vorgehen kann. Es ist notwendiger Bestandteil jeder ,verstehenden' Sozialwissenschaft im Sinne von Max Weber. Zunächst werden dieses Prinzip und seine Rolle im Rahmen der ökonomischen Theoriebildung diskutiert. Dabei wird auch auf die Sinnhaftigkeit einer Mikrofundierung für die makroökonomische Theorie eingegangen. Danach wird aufgezeigt, welche Rolle dieses Prinzip auch in Konzepten eingeschränkter Rationalität spielt. Der Beitrag schließt mit einigen Bemerkungen zur Behandlung der Probleme des freien Willens sowie der Willensschwäche im Rahmen des ökonomischen Verhaltensmodells.

Schlagwörter: Rationalität, Eigeninteresse, mikroökonomische Fundierung, begrenzte Rationalität

\section{The Weak Rationality Principle as the Basis of Every Understanding Social Science}

The weak rationality principle is not an empirical statement but a heuristic rule for how to proceed in social sciences. It is a necessary ingredient of any 'understanding' social science in the Weberian sense. In this paper, first this principle and its role in economic theorizing are discussed. It is also explained why it makes sense to use a micro-foundation and, therefore, to employ the rationality assumption in economic models. Then, we discuss whether the anomalies of individual behaviour as highlighted in modern behavioural economics impair the applicability of the weak rationality principle. This is not the case. We conclude with some remarks on handling the problems of 'free will' as well as 'weakness of the will' within the economic approach.

Keywords: Rationality, Self Interest, Micro-Foundation, Bounded Rationality

Beitrag eingereicht am 15.10.2012; nach verdecktem Gutachterverfahren überarbeitete Fassung angenommen am 10.07.2013.

Prof. Dr. Dr. h.c. Gebhard Kirchgässner, Universität St. Gallen, Institut für Außenwirtschaft und Angewandte Wirtschaftsforschung (SIAW-HSG), Bodanstrasse 8, CH-9000 St. Gallen, Gebhard.Kirchgaessner@unisg.ch, Forschungsschwerpunkte: Politische Ökonomie, Finanzwissenschaft, Umwelt- und Ressourcenökonomik, Angewandte Ökonometrie, methodische Grundlagen der Wirtschafts- und Sozialwissenschaften. Dieser Beitrag ist aus einer früheren englischen Fassung hervorgegangen (vgl. Kirchgässner 2013). Für Anregungen und Hinweise danke ich einem anonymen Gutachter. 


\section{1. $\quad$ Einleitung}

Es dürfte kaum in Frage gestellt werden, dass der Ansatz, menschliches Verhalten als ,rationale Auswahl' (aus Alternativen) bzw. als ,rationales Verhalten' zu erklären, in der Volkswirtschaftslehre unter allen Sozialwissenschaften das größte Gewicht hat. Zwar wird er z.B. auch in der Politikwissenschaft oder der Soziologie eingesetzt, aber dort ist er weit weniger dominant und auch stärker umstritten. Die Spannweite der Varianten dieses Ansatzes geht von der strikten Anwendung der Erwartungsnutzentheorie, wie sie von Neumann und Morgenstern (1944) entwickelt wurde, über Konzepte der vollen und der beschränkten Rationalität im Sinne von Simon (1955) und die Annahme, dass Rationalität schlicht bedeutet „dass die Akteure nicht dumm sind“ (Lagueux 2004: 31) bis zum schwachen Rationalitätsprinzip, welches in dieser Arbeit vorgestellt wird. Entlang dieser Linie nimmt der empirische Gehalt der Rationalitätsannahme ab: Er ist am höchsten für die Erwartungsnutzentheorie und null (oder zumindest ganz nahe bei null) für das schwache Rationalitätsprinzip.

Das Rationalitätsprinzip hat zunächst einen deskriptiven Charakter; es wird verwendet um zu erklären, wie sich Individuen verhalten bzw. wie sie handeln. Gelegentlich wird es jedoch auch als normatives bzw. präskriptives Prinzip angesehen: Es zeigt, wie rationales Verhalten aussehen sollte; es bietet eine Referenz für rationales Verhalten. Dies gilt insbesondere für die von Neumann-Morgenstern Variante der Erwartungsnutzentheorie, die z.B. im Capital-Asset-Pricing-Modell der Finanzmarkttheorie angewendet wird, aber auch für die Bayesianische Entscheidungstheorie. ${ }^{1}$ Eine solche normative Verwendung ist jedoch umso weniger möglich, je geringer sein empirischer Gehalt ist bzw. je schwächer die Rationalitätsannahme ist. Das schwache Rationalitätsprinzip, wie es hier vorgestellt wird, ist für präskriptive Zwecke unbrauchbar. Wir diskutieren hier daher nur die positive Variante dieses Prinzips. ${ }^{2}$

Wann immer die Rationalitätsannahme diskutiert wird, stellt sich die Frage, ob man sie in einem strikt instrumentellen (formalen) Sinn als Zweckrationalität (im Sinne von Max Weber) verstehen oder ob man ihr im Sinne einer Wertrationalität zusätzlich auch einen materiellen Inhalt zuschreiben soll: ob lediglich die Mittel rational in Bezug auf ein zu erreichendes Ziel gewählt werden (bzw. gewählt werden sollten), unabhängig davon, worin dieses Ziel besteht, oder ob diese Ziele selbst rational gerechtfertigt sein sollten. ${ }^{3}$ So hat z.B. Habermas (1964) im ,Positivismusstreit in der deutschen Soziologie in den sechziger Jahren des vergangenen Jahrhunderts Albert (1964) einen „positivistisch halbierten Rationalismus“ vorgeworfen, weil dieser auf der Dichotomie zwischen Tatsachenbehauptungen und Werturteilen bestand, die Habermas mit seiner Konsensustheorie der Wahrheit in Frage stellte.

\footnotetext{
$1 \quad$ Vgl. hierzu Binmore (2009: 1f.), wonach Entscheidungen gemäß der Bayesianischen Theorie in den Wirtschaftswissenschaften häufig mit rationalen Entscheidungen gleichgesetzt werden, obwohl selbst der Vater der Bayesianischen Entscheidungstheorie, Leonard Savage, eingeräumt habe, dass diese Theorie nur einen sehr begrenzten Anwendungsbereich hat.

2 Bezüglich der präskriptiven und deskriptiven Verwendung der Rationalitätsannahme vgl. auch Sen (1987).

$3 \quad$ Nozick (1993: 133) weist darauf hin, dass jedes reichere Konzept von Rationalität instrumentelle Rationalität einschließt. Er selbst argumentiert für ein solch reicheres Konzept.
} 
Unterstellt man eine derartige substantielle Version der Rationalität, so kommt zum präskriptiven Charakter dieser Annahme eine ethische Dimension: Wenn Ziele gerechtfertigt sind, man diese anstreben könnte und dies nicht zu tun irrational wäre, dürfte ein solches Verhalten nicht nur als wenig klug eingeschätzt werden, sondern man sollte es auch aus moralischen Gründen unterlassen. Die positive Ökonomik akzeptiert jedoch die Dichotomie zwischen Tatsachenbehauptungen und Werturteilen, zwischen Sein und Sollen, wie sie auf David Hume (1978/1739) zurückgeht. Deren wesentliche Konsequenz ist, dass es nicht möglich ist, Werturteilen die gleiche wissenschaftliche Absicherung zu geben wie Tatsachenbehauptungen. Damit kommt dem Rationalitätsprinzip auch keine ethische bzw. moralische Dimension zu.

Die Ökonomik folgt somit dem klassischen Modell, ,dass sich Deliberation immer auf Mittel, nie auf Ziele bezieht“", wie Searle (2001: 5) formuliert hat, und verwendet daher in aller Regel eine formale Version der Rationalitätsannahme. Die philosophische Idee dahinter, die aus der Aufklärung stammt, ist die Autonomie der Person bzw., in ökonomischer Terminologie, die ,Konsumentensouveränität‘: Die Individuen sind darin frei, sich ihre eigenen Werte zu wählen, und niemand hat das Recht, ihnen vorzuschreiben, wie ihre Präferenzen auszusehen haben. ${ }^{4}$ Aber auch dann besteht ein weiter Spielraum für unterschiedliche Konzepte der Rationalität. Diese Unterschiede betreffen insbesondere (i) den Inhalt (und die Struktur) der individuellen Nutzenfunktion sowie (ii) die Information, die dem Individuum zur Verfügung steht und wie sie von ihm bzw. ihr verwendet wird. Während letzteres im Wesentlichen im Rahmen der Konzepte eingeschränkter Rationalität thematisiert wird, wie sie von Simon (1955) und Heiner (1983) entwickelt wurden und heute vor allem von Selten (2001) (vgl. auch Harstad/Selten 2013) vertreten wird, dreht sich ersteres vor allem um die Annahme des Eigeninteresses.

Jede starke Version des Rationalitätsprinzips, die empirisch überprüft wurde, wurde verworfen. Dies wird besonders deutlich, sobald empirische Ergebnisse der Spieltheorie betrachtet werden (vgl. hierzu z.B. Selten 1990). Laborexperimente zeigen, dass die Menschen weder den Voraussagen der nicht-kooperativen noch jenen der kooperativen Spieltheorie folgen; und sie widersprechen insbesondere der Annahme des Eigeninteresses als ausschließliche Motivation, die in diesen Modellen verwendet wird. Daher werden heute durch die Ansätze begrenzter Rationalität nicht nur die Informationsprobleme aufgegriffen, sondern auch die Annahmen über die Motivation der handelnden Individuen.

Die empirische Zurückweisung starker Rationalitätskonzepte geht sogar noch weiter. Sogar in Situationen, die (nach traditioneller Auffassung) rationalem Verhalten weitgehend entgegenkommen, weichen die Individuen häufig (und konsistent) davon ab. Beginnend mit dem Aufzeigen des Allais-Paradoxes im Jahr 1952 wurden viele Anomalien des ökonomischen Verhaltensmodells entdeckt, von denen sich einige auf die

4 Dies impliziert freilich nicht, dass niemand das Recht hätte, den autonomen Individuen zu erklären, wie sie sich zu verhalten haben; in den Wirtschaftswissenschaften wird nicht infrage gestellt, dass die Gemeinschaft (bis zu einem gewissen Grad) das Recht besitzt, Regeln des gesellschaftlichen Zusammenlebens festzulegen und damit den Verhaltensspielraum der Individuen zu beschränken. 
Finanzmärkte beziehen. Dabei wurde früher (und wird auch heute noch häufig) davon ausgegangen, dass diese Märkte nicht-rationales Verhalten der Akteure recht streng bestrafen, weshalb den Akteuren kaum etwas anderes übrig bleibt, als sich im Sinne der starken Rationalitätsannahme zu verhalten. Dennoch treten auf diesen Märkten z.B. Montagseffekte, Überschussvolatilität und der Fluch des Gewinners auf (vgl. z.B. die entsprechenden Kapitel in Thaler 1992). Auch hat die große Finanz- und Wirtschaftskrise der vergangenen Jahre das Vertrauen in die Effizienz dieser Märkte - und damit das Vertrauen darein, dass die Akteure sich auf ihnen zumindest so verhalten, als ob sie dem Rationalitätspostulat folgten - stark erschüttert. Und schließlich verwerfen Tests, mit denen überprüft wird, ob die Individuen rationale Erwartungen im Sinn von Muth (1961) haben, diese Hypothese regelmäßig.

Dies impliziert jedoch nicht, dass solche Varianten des Rationalitätsprinzips generell nicht angewendet werden sollten. Um eine Theorie zurückzuweisen, genügt es nicht, dass diese Theorie empirisch falsifiziert ist, sondern man benötigt auch eine alternative Theorie, die für den gewünschten Zweck, besser ${ }^{6}$ geeignet erscheint. Bezüglich der Annahme rationaler Erwartungen ist zu berücksichtigen, dass dies die sparsamste Annahme ist, makroökonomische Modelle zu konstruieren, die nicht implizieren, dass die Individuen konsistent bzw. auf Dauer in gleicher Weise getäuscht werden können. Auch wenn die Annahme rationaler Erwartungen in der empirischen Analyse häufig verworfen wurde (vgl. hierzu z.B. Kirchgässner 1993) und obwohl es Evidenz für die Existenz von Geldillusion gibt (vgl. z.B. Fehr/Tyran 2001), macht es z.B. wenig Sinn, alternative wirtschaftspolitische Strategien unter der Annahme zu entwickeln, dass auch längerfristige Geldillusion existiert, d.h. dass die betroffenen Wirtschaftssubjekte diesbezüglich nicht lernen.

In dieser Arbeit wird demgegenüber die schwächstmögliche Version des Rationalitätsprinzips betrachtet. Sie kann in etwa wie folgt formuliert werden: „Für jede beobachtbare Handlung ist eine Erklärung möglich, die diese als rationales Verhalten erklärt.“ Im Gegensatz zu den strengeren Versionen kann dieses Prinzip durch empirische Evidenz nicht zurückgewiesen werden: Es stellt eher eine methodische Anleitung als eine empirische Behauptung dar. Dennoch ist es, wie noch zu zeigen sein wird, ein grundlegender und notwendiger Bestandteil jeder verstehenden Sozialwissenschaft im Sinne von Max Weber. Selbstverständlich können Sozialwissenschaften auch ohne Einbeziehung dieses Prinzips entwickelt werden, aber dann sind es keine verstehenden Sozialwissenschaften in diesem Sinn.

Andere grundsätzliche Bestandteile einer verstehenden Sozialwissenschaft sind Annahmen über die Ziele der Individuen sowie über die ihnen verfügbare Information. Zusammen ergeben sie das ökonomische Verhaltensmodell. All dies gründet auf dem Konzept des methodologischen Individualismus, d.h. auf der Annahme, dass es ausschließlich die einzelnen Individuen, die Personen, sind, die handeln, auch wenn sie ,gemeinsame Handlungen' durchführen. Daraus ergibt sich eine Mikrofundierung für die Wirtschaftswissenschaften, aber auch für andere Theorien der Gesellschaft. Andere Konzepte für die Entwicklung sozialwissenschaftlicher Theorien, die keine derartige Mikrofundierung beinhalten, benötigen keine Rationalitätsannahme.

Im Folgenden werden zunächst das schwache Rationalitätsprinzip und seine Verwendung in der ökonomischen Theorie dargestellt (Abschnitt 2). Dabei wird auch darauf 
eingegangen, warum es Sinn macht, diese Theorien und Modelle auf eine Mikrofundierung zu stellen und damit die Rationalitätsannahme zu verwenden. Anschließend wird diskutiert, inwieweit die von der Verhaltensökonomie aufgezeigten Verhaltensanomalien die Anwendbarkeit des schwachen Rationalitätsprinzips in Frage stellen (Abschnitt 3). Die Arbeit schließt mit einigen Bemerkungen zur Problematik des freien Willens bzw. der Willensschwäche und ihrer Behandlung im Rahmen des ökonomischen Verhaltensmodells (Abschnitt 4).

\section{Das Rationalitätsprinzip}

In ökonomischen Analysen wird unterstellt, dass die Individuen Intentionen haben, dass ihr Handlungsspielraum beschränkt ist und dass sie mehr oder weniger korrekte und umfassende Informationen sowohl über ihre Handlungsmöglichkeiten als auch über deren Konsequenzen haben. ${ }^{5}$ Letzteres kann von vollständiger Information bis zur totalen Ignoranz gehen. In diesem Rahmen wird Handeln als rationale Auswahl aus den vorhandenen Alternativen bzw. - in der Sprache der Ökonomen - als ,Nutzenmaximierung unter Nebenbedingungen bei Unsicherheit' interpretiert. Dies ist ein konsequentialistischer Ansatz: Die Individuen beurteilen die ihnen bekannten Handlungsalternativen anhand deren erwarteter Konsequenzen (vgl. hierzu auch Sen/Williams 1982).

Für die Entscheidung eines Individuums zwischen den ihm zur Verfügung stehenden Alternativen sind im Rahmen des ökonomischen Verhaltensmodells zwei Punkte wichtig: Die Eigenständigkeit der Entscheidung und ihre Rationalität. Eigenständigkeit bedeutet, dass das Individuum entsprechend seinen eigenen Präferenzen (und nicht entsprechend den Präferenzen anderer) handelt. Selbstverständlich kann es in seinen Präferenzen auch die Interessen anderer mitberücksichtigen; im Extremfall kann es missgünstig, neidisch, oder auch altruistisch sein. ${ }^{6}$

Der zweite Punkt ist die Rationalität der Entscheidung. Wie in der Einleitung bereits ausgeführt wurde, kann das schwache Rationalitätsprinzip wie folgt formuliert werden: „Für jede beobachtbare Handlung ist eine Erklärung möglich, die diese als rationales Verhalten erklärt." Dies ist eine gemischte Aussage, die eine Allaussage mit einer Existenzbehauptung verknüpft. Solche Aussagen sind weder verifizierbar noch falsifizierbar und können daher auch nicht durch empirische Evidenz zurückgewiesen werden; sie können deshalb als ,metaphysisch ${ }^{6}$ betrachtet werden. ${ }^{7}$ Dennoch können sie, worauf Watkins $(1957,1958,1975)$ mehrfach hingewiesen hat, in den Wissenschaften eine wesentliche Funktion erfüllen, wenn sie als heuristische Regel verstanden werden: „Wo immer Du ein Verhalten beobachtest, versuche, es als rationales Handeln zu erklären!“

$5 \quad \mathrm{Zu}$ einer ausführlichen Darstellung des ökonomischen Verhaltensmodells vgl. Kirchgässner (2008: insbesondere 12ff.).

$6 \quad$ In der Terminologie von Sen (1985: 347; 2007: 17) wird „Wahl nach den eigenen Zielvorstellungen“ (self-goal choice) unterstellt. Im Gegensatz zu seiner Auffassung schließt dies jedoch kein Verhalten und auch keine Motivation aus.

7 Zur Diskussion solcher gemischter (,all-and-some“) Aussagen vgl. neben Watkins (1957) auch Pähler (1986: 24, 75ff.). 
Bezogen auf das Verhalten des Individuums bedeutet Rationalität hier somit nicht, dass dieses in jedem Augenblick optimal handelt, dass es also gleichsam wie ein wandelnder Computer durch die Welt schreitet, der immer die beste aller vorhandenen Möglichkeiten blitzschnell ermittelt. Dieses Zerrbild des homo oeconomicus, das bis heute in vielen Lehrbüchern der mikroökonomischen Theorie zu finden ist und das mit Recht immer wieder Kritik herausgefordert hat, entspricht nicht der modernen Interpretation des ökonomischen Verhaltensmodells. ${ }^{8}$ Rationalität bedeutet hier lediglich, dass das Individuum, wenn es seinen Intentionen folgt, prinzipiell in der Lage ist, gemäß seinem relativen Vorteil zu handeln, d.h. seinen Handlungsraum abzuschätzen und zu bewerten, um dann entsprechend zu handeln. ${ }^{9}$ Dabei ist zu berücksichtigen, dass das Individuum sich immer unter unvollständiger Information entscheiden muss und dass die Beschaffung zusätzlicher Information Kosten verursacht. Auch muss es häufig unter Zeitdruck entscheiden. Kosten zur Informationsbeschaffung wird das Individuum vor allem dann auf sich nehmen, wenn es eine relevante Veränderung seines Handlungsraumes (und/oder seiner Präferenzordnung) registriert und daher eine neue Abschätzung und Bewertung seiner Handlungsmöglichkeiten vornehmen muss. Auf eine solche Veränderung reagiert ein rationales Individuum, systematisch', d.h. nicht zufällig oder willkürlich, aber auch nicht dadurch, dass es sich unabhängig von diesen Veränderungen streng an vorgegebene Regeln hält. ${ }^{10}$

Damit kann dieses Verhalten durch Setzung von Anreizen systematisch beeinflusst werden, wobei sich solche Anreize im Wesentlichen aus Veränderungen des Handlungsraums der Individuen (der Restriktionen) ergeben. In diesem Konzept verschwindet daher der philosophisch bedeutsame und häufig diskutierte Unterschied zwischen Verhalten und Handeln: Verhalten von Individuen wird erklärt, indem unterstellt wird, dass sie rational handeln. ${ }^{11}$ Damit sind auch Prognosen von Verhaltensänderungen als Reaktion auf Veränderungen des Handlungsspielraums möglich.

Weber nimmt eine ähnliche, wenn nicht die gleiche Position ein, wenn er über soziales Verhalten schreibt:

„,Handeln‘ soll dabei ein menschliches Verhalten (einerlei ob äußeres oder innerliches Tun, Unterlassen oder Dulden) heißen, wenn und insofern als der oder die Handelnden mit ihm einen subjektiven Sinn verbinden. ,Soziales' Handeln aber soll ein solches Handeln heißen, welches seinem von dem oder den Han-

Zur Kritik an diesem Konzept der Rationalität vgl. auch Arrow (1986).

9 Eine ähnliche Formulierung des ,Rationalprinzips“, die er jedoch als „nur grob und provisorisch“ bezeichnet, gibt z.B. Watkins: „Ein Individuum ist in eine bestimmte objektive Problemsituation hineingestellt. Es hat bestimmte Ziele (Bedürfnisse, Präferenzen) oder vielleicht ein einziges Ziel, und es schätzt die seine Problemsituation ausmachenden Tatsachen ab (wobei es sie falsch einschätzen kann). Nach dem Rationalprinzip wird dieses Individuum so handeln, wie es seinem Ziel oder seinen Zielen und seiner Einschätzung der Situation ,angemessen' ist"“ (1978: 35). Bei dem Terminus ,angemessen` bezieht er sich ausdrücklich auf Popper (1967).

10 Zur Diskussion dieser beiden Arten ,irrationalen“ Verhaltens vgl. Becker (1962) sowie, unter Bezug darauf, Elster (1979: 137ff).

11 Vgl. hierzu auch Kirchgässner (1985). Freilich ist diese Auffassung nicht unbestritten. Eine Gegenposition findet sich insbesondere bei solchen Autoren, die mit dem Handlungsbegriff einen moralischen Anspruch verbinden, wie z.B. Patzak (1984). 
delnden gemeinten Sinn nach auf das Verhalten anderer bezogen wird und daran in seinem Ablauf orientiert ist." (1922: 1)

Mit anderen Worten wird im Rahmen des ökonomischen Verhaltensmodells unterstellt, dass sich Individuen an veränderte Umweltbedingungen entsprechend ihrer Zielvorstellungen (Präferenzen) in systematischer und damit vorhersagbarer Weise anpassen, wobei sich solche Veränderungen sowohl durch Handeln anderer Individuen, z.B. durch politische Maßnahmen, als auch durch Veränderungen der ,natürlichen` Bedingungen ergeben können. Kliemt formuliert dies als Prinzip folgendermaßen:

„Alles intentionale menschliche Verhalten ist als präferenzgeleitetes, individuelles Anpassungsverhalten zu erklären.“ (1984: 17)

Wissenschaftslogisch dürfte das dem ökonomischen Verhaltensmodell zugrunde liegende schwache Rationalitätsprinzip für die Sozialwissenschaften einen ähnlichen Stellenwert haben wie das ,Kausalitätsprinzip“ in den Naturwissenschaften. ${ }^{12}$ So wie dort das Reden über (Natur-)Gesetze erst dann möglich ist, wenn man das Kausalitätsprinzip akzeptiert, ist in den Sozialwissenschaften das Verstehen menschlichen Handelns erst möglich, wenn man die im ökonomischen Verhaltensmodell angelegte Unterscheidung zwischen Präferenzen und Restriktionen (Zielen und Mitteln) akzeptiert und außerdem davon ausgeht, dass die Individuen die ihnen zur Verfügung stehenden Mittel (rational) zur Erreichung ihrer Ziele einsetzen, wobei natürlich die subjektiven Perzeptionen eine wichtige Rolle spielen.

Interessanterweise arbeitet nicht nur die ,Neue Wirtschaftsgeschichte', wie sie z.B. von North (1981) vertreten wird, sondern auch die traditionelle, ,verstehende ${ }^{6}$ Geschichtswissenschaft (implizit) mit genau diesem ,ökonomischen' Verhaltensmodell und damit auch mit dem dahinter stehenden Rationalitätsprinzip, obwohl es zunächst so scheint, als ob Geschichtswissenschaft und (theoretische) Ökonomie methodisch sehr weit auseinander liegen würden. ${ }^{13}$ In diesem Zusammenhang schreibt z.B. Watkins über das (schwache) Rationalitätsprinzip:
„Aber das Prinzip kann auch in die Form einer methodologischen Regel gefasst werden, die den Historikern und anderen Forschern, die sich mit dem Verhalten der Menschen befassen, einschärft, das Prinzip nicht notwendigerweise qua fak- tisches Postulat als wahr zu akzeptieren, sondern so, als ob es wahr sei, vorzu- gehen. In dieser letzten Form (...) bedeutet es vor allem, dass, um eine Konjek- turalerklärung einer vergangenen Handlung zu liefern, ein Entscheidungsplan

12 Zum Rationalitätsprinzip vgl. auch Popper (1967); Tietzel (1981: 131ff.); Abel (1983: 133ff.); sowie für eine etwas andere Position Latsis (1983). Auch beim Kausalitätsprinzip handelt es sich um eine gemischte Aussage, vgl. Watkins (1958: 348). Natürlich kann man das Kausalitätsprinzip auch anders auffassen. Zur Bedeutung des Kausalitätsprinzips vgl. z.B. Stegmüller (1960) oder Bunge (1959). Außerdem gilt die Analogie zwischen Rationalitätsprinzip und Kausalitätsprinzip nur bedingt. Während man z.B. das Rationalitätsprinzip auch normativ auffassen kann, gilt dies für das Kausalitätsprinzip trivialerweise nicht.

13 Dieser Anschein wird u.a. durch den von Menger (1883) ausgelösten ,Methodenstreit ${ }^{\star}$ zwischen $^{2}$ dessen theoretischer (österreichischer) Schule und Schmollers historischer Schule der Nationalökonomie geliefert, vgl. hierzu z.B. Schumpeter (1965: 994ff.). - Zur Anwendung des Rationalmodells in der Geschichtswissenschaft vgl. auch Frings (2007). 
postuliert werden muss, der eine praktische Schlussfolgerung zulässt, deren natürliches Ergebnis diese Handlung sein könnte.“ (1978: 79)

Hier wird deutlich, dass das schwache Rationalitätsprinzip keine empirische Aussage beinhaltet und damit weder verifiziert noch falsifiziert werden kann. Wann immer die Handlung eines Individuums beobachtet wird, kann sie im obigen Sinn rational sein, selbst wenn wir sie (im Augenblick) nicht verstehen können. Andererseits könnten wir selbst dann, wenn wir eine in diesem Sinne ,rationale Erklärung für jedes beobachtete Handeln hätten, nie sicher sein, dass dies für jede menschliche Handlung gilt. Insofern ist das schwache Rationalitätsprinzip, wie bereits erwähnt wurde, eine metaphysische Aussage. Sein Zweck ist nicht, uns etwas über die Realität mitzuteilen, sondern es ist ein heuristisches Prinzip, welches uns methodische Anleitung dafür gibt, wie wir in den Sozialwissenschaften vorgehen können. Es sagt uns, dass wir immer dann, wenn wir menschliches Verhalten erklären wollen, versuchen sollten, es als rationales Handeln der Individuen bzw. als Ergebnis rationaler Entscheidungen zu interpretieren. ${ }^{14}$

Dies steht in einem gewissen Gegensatz zu Poppers Standpunkt zum Rationalitätsprinzip. Einerseits gibt es bestenfalls eine geringe Meinungsverschiedenheit über den Inhalt des Prinzips und seine Bedeutung für die Sozialwissenschaften. Er sieht im ökonomischen Ansatz „eine rein objektive Methode in den Sozialwissenschaften (...), die man wohl als objektiv-verstehende Methode oder als Situationslogik bezeichnen kann" (1962: 120f.). In seiner „Objektiven Erkenntnis“ bezeichnet er dieses Vorgehen auch als „Situationsanalyse“ (1972: 199). Er definiert das Rationalitätsprinzip auch als „das Prinzip, dass man der jeweiligen Situation angemessen reagiert" (1962: 121). Andererseits gibt es einen Dissens betreffend des Status dieses Prinzips. Obwohl Popper anerkennt, dass es „klarerweise ein nahezu leeres Prinzip“ (1967: 359) ist, glaubt er, dass es etwas empirischen Gehalt hat und damit - zumindest im Prinzip - testbar ist. Unter Berufung auf prima facie-Evidenz stellt er dann fest: „Das Rationalitätsprinzip ist falsch“ (Popper 1967: 361). Dennoch anerkennt er dessen heuristischen Wert. ${ }^{15}$

Wird dieses Verhaltensmodell verwendet, um Handlungen von Menschen zu verstehen, verschwindet die Differenz zwischen ,Verstehen' und ,Erklären': Ich kann menschliches Handeln nur verstehen, wenn ich es mit Hilfe eines solchen Modells rationalen Verhaltens erklären kann. Ähnlich formuliert Weber, der üblicherweise der ,verstehenden Sozialwissenschaft ${ }^{\star}$ zugerechnet wird. Er sieht in der Soziologie „eine Wissenschaft, welche soziales Handeln deutend verstehen und dadurch in seinem Ablauf und seinen Wirkungen ursächlich erklären will““ (1922: 1). ${ }^{16}$ Dies entspricht auch unserem üblichen Sprachgebrauch von ,Verstehen': Auch hier denken wir in

14 Vgl. hierzu auch Vanberg (2004: 3): „Interpretiert als ein heuristisches Prinzip sagt uns das Rationalitätsprinzip, wie wir bei der Erklärung zielgerichteten Handelns vorgehen sollen. Es legt nahe, dass wir versuchen sollten, solche Handlungen in Bezug auf die Ziele und Vorstellungen der Akteure zu erklären, und dass wir dies unter der Annahme tun sollten, dass ihre Ziele und Vorstellungen im Zeitpunkt der Entscheidung konsistent sind.“

15 Zur Diskussion und Kritik von Poppers Konzeption der Sozialwissenschaften vgl. Vanberg (1975: 109ff.), Schmid (1979a; 1979ba), Hands (1985) sowie Lagueux (2010: 103ff.).

16 Zur Position von Weber vgl. z.B. Angehrn (1983). 
Kategorien von Motiven (Präferenzen), Mitteln (Restriktionen) und beschränkter Information. ${ }^{17}$

Mit Nozick (1993: 133ff.) kann man das schwache Rationalitätsprinzip auch als instrumentelle Rationalität verstehen. Er vertritt die Auffassung, das instrumentelle Rationalität völlig unbestritten ist und jeder weitergehenden Rationalitätskonzeption zugrunde liegt. Instrumentelle Rationalität und schwaches Rationalitätsprinzip sind freilich nur dann deckungsgleich, wenn ich im Rahmen der instrumentellen Rationalität beliebige Annahmen über die subjektiven Zustände zulasse: sowohl über die Präferenzen der Beteiligten als über ihre Einschätzung der jeweiligen Situation. Instrumentelle Rationalität kann jedoch auch ,objektiv' verstanden werden, d.h. man kann fragen, ob eine bestimmte Handlung tatsächlich dazu geeignet war, ein bestimmtes, vom Handelnden angestrebtes Ziel zu erreichen. Dann wird die Frage, ob sich jemand in einer bestimmten Situation instrumentell rational verhalten hat, zu einer empirisch überprüfbaren Hypothese.

Gemäß dem schwachen Rationalitätsprinzip mag sich dagegen ein Individuum rational verhalten, selbst wenn es - entsprechend unserer üblichen Vorstellungen - recht seltsame Dinge tut. Jemand, der z.B. schizophren ist und glaubt, dass er eine andere Person sei oder sich in einer vollständig anderen Situation befindet, mag in diesem Sinn ,rational' sein, gegeben seine (total falsche) Vorstellung von der Realität. Wir können ihn auch nur dann verstehen, wenn wir seine Perzeption aufgreifen und dann fragen, weshalb er sich in dieser oder jener Weise verhalten haben könnte. Sobald wir „Warum" fragen, nehmen wir üblicherweise an, dass ein Akteur ,Gründe' für sein Verhalten hatte, wie merkwürdig diese auch immer sein mögen, womit wir implizit das schwache Rationalitätsprinzip anwenden.

Die einzig ,starke' Annahme in diesem Konzept ist die Annahme der Konsistenz (im Augenblick der Entscheidung): Es wird unterstellt, dass die Präferenzen insofern vollständig sind, als vom Individuum angenommen wird, dass es prinzipiell in der Lage ist, all jene Handlungsalternativen, die es als Möglichkeit in Betracht zieht, einzuschätzen, und dass diese Bewertungen transitiv sind. Ohne diese Annahme können wir nicht ,verstehen', was ein Individuum tut. Aber auch diese Annahme ist nicht testbar, solange wir nicht unterstellen, dass die Präferenzen über die Zeit hinweg mehr oder weniger konstant sind. Handlungen zu verschiedenen Zeitpunkten, die inkonsistent erscheinen mögen, können konsistent sein, falls das Individuum nur über eine begrenzte Informationsverarbeitungskapazität verfügt oder falls sich seine Präferenzen geändert haben. Darüber hinaus kann auch Lernen (oder Vergessen) das Verhalten des Individuums so beeinflussen, dass es nach außen als inkonsistent erscheint. Konsistenz über die Zeit hinweg ist eine (notwendige) Voraussetzung für eine Analyse mit Hilfe des Ansatzes aufgedeckter Präferenzen, aber sie ist kein notwendiges Element der Rationalitätsannahme (vgl. Binmore 2009: 9ff.; sowie Sudgen 1985).

Die Anwendung des ökonomischen Verhaltensmodells ist bei weitem nicht der einzige Weg, wie die Sozialwissenschaften vorgehen können. Der methodologische Individualismus ist genauso wenig ein notwendiges Element jeder Sozialwissenschaft wie die 
Rationalitätsannahme. Dementsprechend bedarf auch nicht jede Sozialtheorie einer Mikrofundierung. ${ }^{18}$ Man kann Makrotheorien entwickeln, die z.B. Eigenschaften sozialer Systeme und die Beziehungen zwischen diesen untersuchen und die dabei weder einer Mikrofundierung bedürfen noch das schwache Rationalitätsprinzip bemühen müssen. Dies gilt auch für die Volkswirtschaftslehre, die ja weniger am Verhalten einzelner Individuen als vielmehr am Verhalten größerer Gruppen von Individuen, so genannter ,Aggregate' interessiert ist, wie z.B. der Konsumenten, der Unternehmer oder auch der Wähler. Nicht das Verhalten eines bestimmten einzelnen Individuums ist für uns interessant, sondern wir suchen nach dem ,typischen 'Verhalten, d.h. nach Regelmäßigkeiten im Verhalten aller oder zumindest einer Mehrheit der betrachteten Individuen in der jeweils untersuchten Gruppe. ${ }^{19}$ Die Mikrotheorie bietet hier (nur) die Basis, um Makrophänomene erklären zu können.

Dies ist kein Widerspruch, wie es zunächst scheinen könnte. Werden nämlich durch eine Veränderung einer bestimmten Makrovariablen die Rahmenbedingungen für das Handeln aller Individuen bzw. einer bestimmten Gruppe in ähnlicher Weise beeinflusst, kann man davon ausgehen, dass deren Reaktion zwar nicht unbedingt in jedem Einzelfall, aber im Durchschnitt jene Regelmäßigkeit aufweist, welche aus dem individuellen Entscheidungskalkül heraus erklärt werden kann. So wird z.B. auf eine Erhöhung des Benzinpreises - ceteris paribus - nicht jeder einzelne Autofahrer mit einer Einsparung von Benzin reagieren. Wichtig für den ökonomischen Zusammenhang ist ausschließlich, dass der Durchschnitt aller Verbraucher mit einer Einsparung reagiert, so dass die Erhöhung der Preise insgesamt eine Reduktion der nachgefragten Menge bewirkt. Dieses Verhalten, wie es sich tatsächlich auch im Anschluss an die großen Benzinpreiserhöhungen der Jahre 1973/74 und 1979/80 ergeben hat und wie es auch im Jahr 2008 wieder beobachtet werden konnte, kann (unter einigen zusätzlichen ,schwachen' Annahmen) für den ,typischen' Verbraucher aus dem individuellen Optimierungskalkül der Konsumtheorie abgeleitet werden.

Ob eine Mikrofundierung für ökonomische (Makro-)Theorien erforderlich ist, wurde z.B. in den siebziger Jahren des vergangenen Jahrhunderts diskutiert (vgl. hierzu z.B. Ramser 1987: 8ff.; Machlup 1960; Schlicht 1977). Auf Plausibilitätsargumenten beruhende Makrorelationen wurden akzeptiert, solange sie mit Hilfe ökonometrischer Methoden statistisch abgesichert schienen. Wirtschaftspolitische Maßnahmen wurden im Vertrauen auf die Existenz und Stabilität dieser Beziehungen getroffen.

Das berühmteste Beispiel für eine solche Beziehung dürfte die modifizierte PhillipsBeziehung sein, jene (behauptete) langfristige Beziehung zwischen Inflation und Arbeitslosigkeit. Sie wurde zuerst von Phillips (1958) als Beziehung zwischen Arbeitslosigkeit und Nominallohnentwicklung, entdeckt' und später zu einer Beziehung zwi-

18 Für eine Diskussion der beiden Soziologien, die eine mit und die andere ohne Mikrofundierung, vgl. Vanberg (1975).

19 So schreibt z.B. Hicks bei der Behandlung des Nachfragegesetzes: „In allen bisherigen Diskussionen haben wir uns mit dem Verhalten eines einzelnen Individuums befasst. Aber die Ökonomie ist letztlich nicht sehr interessiert am Verhalten einzelner Individuen. Sie befasst sich mit dem Verhalten von Gruppen. Eine Untersuchung der individuellen Nachfrage ist nur ein Hilfsmittel zur Untersuchung der Marktnachfrage“ (1939: 34). - Vgl. hierzu auch Hayek (1952: 48ff.) sowie Popper (1967: 142). 
schen Inflation und Arbeitslosigkeit erweitert. Im Jahr 1960 wurde sie von Samuelson und Solow als „Speisekarte der Wirtschaftspolitik“ bezeichnet. Solche, nach ihrem ,Entdecker' benannten ,Phillips-Kurven' wurden für eine ganze Reihe von Ländern ökonometrisch geschätzt, und gestützt auf diese empirische Evidenz glaubte man, durch eine einmalige Erhöhung der Inflationsrate die Arbeitslosenquote dauerhaft senken zu können. ${ }^{20}$

Solche makroökonomischen Beziehungen bzw. die aus ihnen bestehenden makroökonometrischen Modelle sind in vielen Fällen hilfreiche Prognoseinstrumente. Wie Lucas (1976) in seiner berühmten Kritik der ökonometrischen Politikevaluation gezeigt hat, kann sich dies ändern, sobald man versucht, mit ihrer Hilfe die Auswirkungen unterschiedlicher Wirtschaftspolitiken zu simulieren und diejenigen wirtschaftspolitischen Maßnahmen in die Praxis umzusetzen, die zum (nach Ansicht der politischen Entscheidungsträger) ,besten' Ergebnis führen. Dann kann es geschehen, dass eine makroökonomische Relation, die man wirtschaftspolitisch anwenden wollte, verschwindet. Will man z.B. die (statistisch abgesicherte) langfristige Austauschbeziehung zwischen Inflation und Arbeitslosigkeit dadurch ausnützen, dass man durch eine Politik des billigen Geldes die Inflation erhöht, um die Arbeitslosenquote zu senken, so mag dem kurzfristig vielleicht ein Erfolg beschieden sein, aber auf mittlere oder gar längere Frist wird man sich damit abfinden müssen, dass sich zwar die Inflationsrate dauerhaft erhöht, die Arbeitslosenquote aber wieder ihre alte Höhe erreicht oder sogar noch darüber hinaus ansteigt.

Tatsächlich ist die politische (experimentelle) Ausnutzung der Phillips-Kurve gescheitert. In den siebziger und achtziger Jahren stiegen die Preise zusammen mit der Arbeitslosigkeit. Der Grund für diesen Fehlschlag war der Umstand, dass diese Politik auf die Geldillusion der Wirtschaftsakteure vertraute. Eine solche Politik könnte nur dann langfristig erfolgreich sein, wenn es möglich wäre, die Wirtschaftsakteure permanent und systematisch zu täuschen. Dies ist nicht nur wenig plausibel; es widerspricht auch der Rationalitätsannahme des ökonomischen Ansatzes. Heute ist die Notwendigkeit einer Mikrobasis für Makroanalysen in den Wirtschaftswissenschaften weitgehend akzeptiert.

\section{Die Rationalitätsannahme und die Ergebnisse der Verhaltensökonomik}

Wie oben bereits erwähnt wurde, kann das schwache Rationalitätsprinzip weder falsifiziert noch verifiziert werden; es hat einen metaphysischen Charakter, kann aber eine hilfreiche methodische Anleitung darstellen, und oft ist es das auch. Sein empirischer Gehalt ist jedoch null. A priori mag dies kein größeres Problem darstellen, wenn z.B. ein Geschichtswissenschaftler versucht, eine bestimmte historische Episode zu erklären. Aber auch dann benötigt er zusätzliche Annahmen über die Motivation der handelnden Personen sowie über die Information, über welche diese verfügten. Trifft er diese Annahmen falsch, dürfte sein Erklärungsversuch scheitern.

Es gibt jedoch ein sehr viel bedeutenderes Problem, sobald wir allgemeine Hypothesen über menschliches Verhalten sowie insbesondere, wie es in der Ökonomie üblich

20 Vgl. hierzu und zur Kritik daran Ramser (1987), Santomero/Seater (1978) sowie die Diskussion in den Vereinigten Staaten zwischen King/Watson (1994) und Evans (1994). 
ist, über die gesellschaftlichen Auswirkungen individuellen Verhaltens ableiten wollen. In diesem Fall müssen wir ebenfalls, wie jener Historiker, zusätzliche Annahmen über die Motivationen der handelnden Personen sowie über die ihnen zur Verfügung stehende Information treffen. Je stärker diese Annahmen sind, desto höher ist der empirische Gehalt unserer Theorie, desto höher ist aber auch die Wahrscheinlichkeit, dass unsere Theorie oder zumindest einige ihrer Hypothesen am ,Widerstand der Realität scheitern. Wann immer dies der Fall ist, ist es nicht die schwache Rationalitätsannahme, die scheitert; zumindest eine der zusätzlichen Annahmen ist für dieses Scheitern verantwortlich.

Werden solche Tests zum Beispiel mit Hilfe von Experimenten durchgeführt, wird aus deren Ergebnissen oft der Schluss gezogen, dass sich die Individuen nicht rational verhalten bzw. dass das ökonomische Verhaltensmodell, der ,Homo Oeconomicus', gescheitert ist. Manche proklamieren sogar: „Der Homo Oeconomicus ist tot“ (Häring 2001)! Dies ist jedoch ein kategorialer Fehler. In diesen Experimenten wird häufig die in ökonomischen Analysen üblicherweise verwendete Annahme reinen Eigeninteresses, die Motivationsannahme, verworfen, und nicht die Rationalitätsannahme. Auch wenn dies häufig und auch bei hoch reputierten Ökonomen geschieht, sollte man diese zwei Dinge nicht durcheinander bringen. ${ }^{21}$ Es ist offensichtlich, dass die Individuen neben dem reinen Eigeninteresse noch andere Motive haben. Ob die sparsame Motivationsannahme des Eigeninteresses sinnvoll ist, um eine bestimmte Situation zu analysieren, hängt von den konkreten Umständen ab und ist nicht eine Frage, ob reines Eigeninteresse - im engen Sinn - die einzige Motivation ist, der die Individuen folgen. Und es ist noch viel weniger eine Frage der Rationalität.

Es wird offensichtlich, dass dies keine Frage der Rationalität ist, wenn man die Arbeit von Fehr und Schmidt (1999) betrachtet. Um eine Theorie zu entwickeln, die mit den Ergebnissen ihrer Experimente vereinbar ist, modifizieren sie leicht die Nutzenfunktion der Individuen, indem sie Ungleichheitsaversion als zusätzliches Element einführen, und wenden dann das übliche Maximierungsprozedere an. Aus der Perspektive des traditionellen Vorgehens in der Ökonomie, welches rationales Verhalten als Nutzenmaximierung unter Nebenbedingungen interpretiert, ist ihr Vorgehen nichts anderes als die Anwendung dieses Ansatzes mit einer kleinen Modifikation der Nutzenfunktion. Methodisch betrachtet ist dies nur eine geringfügige Abänderung, auch wenn die Konsequenzen für das Erklärungspotential beträchtlich sind.

Es gibt zumindest drei Phänomene, die empirisch gut bestätigt, aber mit der Annahme des (eng verstandenen) Eigeninteresses nicht vereinbar sind: Altruismus, Reziprozität und Commitment. ${ }^{22}$ Folgt man Sen (1977: 209), wonach bei Commitment eine Situa-

21 Vgl. hierzu z.B. auch Hausman und McPherson (1996: 2), die explizit erwähnen, dass in der positiven Ökonomik die Annahme des Eigeninteresses zu derjenigen der Rationalität hinzugefügt wird. Im Gegensatz dazu trägt z.B. Sen (2005: 5) zur Verwirrung bei, wenn er schreibt, dass der Fokus der Rational Choice-Theorie darin besteht, „Rationalität bei der Wahl [der Handlungsalternative; GK] als Maximierung des Eigeninteresses zu charakterisieren.“

22 In der deutschen Übersetzung von Sen (1977) wird Commitment mit ,verantwortliche Verpflichtung“ übersetzt. Inzwischen hat sich jedoch auch in der deutschsprachigen Liternatur „Commitment" als Fachbegriff durchgesetzt, weshalb wir hier diesen Begriff und nicht die Übersetzung verwenden. 
tion vorliegt, in welcher eine Person einen Akt wählt, ,von dem sie annimmt, dass er ihr weniger persönliche Wohlfahrt einbringt als eine ebenfalls offenstehende Alternative", dann geht es in allen drei Fällen darum, dass eine Person sich selbst Kosten auferlegt, die keinen (direkten) positiven Beitrag zu ihrer individuellen Wohlfahrt leisten. Im Falle von Altruismus hat die entsprechende Handlung direkte positive Auswirkungen auf die Wohlfahrt anderer Individuen. Im Fall der Reziprozität können diese Auswirkungen in Abhängigkeit von den vorangehenden Handlungen dieser Individuen positiv oder negativ sein; der wirklich interessante Fall ist derjenige der negativen Reziprozität, wenn jemand Kosten auf sich nimmt, um einen anderen zu bestrafen. ${ }^{23}$ Folgt man der oben gegebenen Definition von Commitment, ist offen, wie die Auswirkungen sind. Eine Person kann sich zu einem Verhalten verpflichtet fühlen, welches Anderen Nutzen stiftet, oder zu einem Verhalten, welches anderen schadet, aber auch zu einem Verhalten, welches keine Auswirkungen auf andere hat. Insofern können Altruismus und Reziprozität als Spezialfälle von Commitment angesehen werden. ${ }^{24}$ Der wesentliche Unterschied betrifft das Vorzeichen des entsprechenden Arguments in der Nutzenfunktion.

Es wurde gezeigt, wie Altruismus, Reziprozität oder moralisches Verhalten in ökonomischen Modellen, die Verhalten als Nutzenmaximierung unter Nebenbedingungen darstellen, repräsentiert werden können. ${ }^{25}$ Solches Verhalten zu formalisieren ist völlig problemlos: Die Aufgabe besteht darin, die Nutzenfunktion entsprechend zu spezifizieren. Dies tangiert in keiner Weise die schwache Rationalitätsannahme. Es gibt aus dieser Perspektive z.B. keinen Widerspruch zwischen Rationalität und moralischem Verhalten. Und der von Sen (1977) beschriebene „Rationalclown“ mag zwar als Clown betrachtet werden, aber nicht wegen seiner Rationalität, sondern weil er eine sehr merkwürdige Nutzenfunktion (mit sehr hoher Diskontrate) hat.

Man kann infrage stellen, ob es angemessen ist, solches nicht durch Eigeninteresse bestimmtes Verhalten in den (üblichen) ökonomischen Ansatz zu integrieren, um damit bei der Formulierung von Rationalwahlmodellen mehr Raum für Präferenzen zu schaffen, die andere betreffen (vgl. hierzu auch Sen 1994: 389). Dass man moralischen Phänomenen im Rahmen der Nutzentheorie Rechnung tragen kann, impliziert nicht, dass es auch angemessen ist, solche Phänomene auf diese Art und Weise zu analysieren. ${ }^{26}$ Aber dies ist eine Frage der Angemessenheit und nicht eine Frage der Möglichkeit. Die Verwendung von Modellen, die mit dem ökonomischen Ansatz arbeiten, erlaubt die Ableitung testbarer Hypothesen darüber, unter welchen Bedingungen mit moralischem Verhalten gerechnet werden kann (vgl. hierzu auch Kirchgässner 1992). Die theoretische und empirische Literatur über die Wahlbeteiligung, die

23 Positive Reziprozität wie z.B. im Fall von Tit-for-Tat kann durchaus im langfristigen Eigeninteresse des Individuums liegen und muss daher der in ökonomischen Modellen üblichen Motivationsannahme nicht widersprechen.

24 Analog zum Commitment kann man auch für die, Wertrationalität‘ im Konzept von Max Weber argumentieren.

25 Für Altruismus vgl. z.B. Andreoni (1988; 1989; 1990), für Reziprozität Fehr/Gächter (1998; 2000) und für moralisches Verhalten Kirchgässner (2002; 2010).

26 So argumentieren z.B. Hausman/McPherson (1996: 53), ,dass manchen moralischen Phänomenen im Rahmen der Nutzentheorie nicht Rechnung getragen werden kann.“ 
seit Riker und Ordeshook (1968) moralisches Verhalten im Rahmen eines traditionellen ökonomischen Ansatzes berücksichtigt, ist hierfür ein gutes Beispiel. ${ }^{27}$ Zudem ist völlig offen, wie eine Formalisierung solchen Verhaltens in einem alternativen Modell aussehen könnte, wenn man nach wie vor eine Mikrobasis für Makrotheorien wünscht und davon ausgeht, dass die Individuen Intentionen haben.

Wie oben ausgeführt wurde, wurden u.a. in Experimenten starke Versionen der Rationalitätsannahme verworfen. Dies geschah wohl zum ersten Mal auf der berühmten Paris Konferenz im Mai 1952, als Savage versuchte, sein Unabhängigkeitsaxiom zu verteidigen. Dieses Axiom ist im Erwartungsnutzenkonzept von von Neumann/Morgenstern (1944) implizit enthalten. Allais (1953) konnte zeigen, dass Savage sein eigenes Axiom verletzte. ${ }^{28}$ In diesem und ähnlichen Fällen sind es bestimmte Versionen der Rationalitätsannahme, die zurückgewiesen werden. Aber dies bedeutet keine Verwerfung des schwachen Rationalitätsprinzips: Es sind sehr starke Versionen dieses Prinzips, die verworfen werden.

In diesen klassischen Experimenten sind die Versuchspersonen vollständig informiert. In anderen Experimenten sowie insbesondere in realen Situationen sind sie dagegen kaum jemals vollständig informiert; in aller Regel ist ihre Information sehr lückenhaft, sei es bezüglich der ihnen zur Verfügung stehenden Alternativen, sei es bezüglich deren möglicher Konsequenzen. Hier greift das Konzept der ,begrenzten` bzw. ,prozeduralen' Rationalität, wie es von Simon (1955) entwickelt wurde (vgl. hierzu auch Simon 1978, 1979; Selten 1990, 2001; Conlisk 1996; Rubinstein 1998; Harstad/Selten 2013). Dort unterscheidet sich das Verhalten erheblich von demjenigen, welches unter ,voller' oder ,substantieller ' Rationalität zu erwarten wäre. Aber selbst dann ist es nicht nur das schwache Rationalitätsprinzip, welches zur Anwendung kommt. Es ist darüber hinaus der ökonomische Ansatz mit seinen grundlegenden Annahmen: dass die Individuen Intentionen haben, dass sie über ein - mehr oder weniger richtiges oder falsches - Bild der Realität verfügen, und dass sie dementsprechend die von ihnen in Betracht gezogenen Handlungsmöglichkeiten bewerten. Auch hier folgen sie dem schwachen Rationalitätsprinzip, indem sie - gegeben ihre Information und ihre Erwartungen - die ihnen am besten erscheinende Handlungsalternative wählen.

Die Situation mag völlig anders aussehen, wenn wir wie in den berühmten Experimenten von Tversky/Kahneman (1981; 1987) ,Framing-Effekte“ beobachten (vgl. hierzu auch Stocké 2002). Hier scheint die oben diskutierte Konsistenzannahme verletzt zu sein. Dies muss jedoch nicht der Fall sein. Wenn zwei unterschiedliche Darstellungen verschiedene Situationen nahelegen, werden auch vernünftige Menschen unterschiedlich reagieren. Es handelt sich hier um ein Problem der Information bzw. der Informationsverarbeitung, nicht aber um eines der Rationalität. Wir befinden uns in einer Situation der begrenzten Rationalität; es besteht keine Veranlassung, hier von Irratio-

$27 \quad$ Dass Green/Shapiro (1994) die Wahlbeteiligung als eines ihrer Beispiele für das Versagen des Rationalwahl-Ansatzes heranziehen, widerspricht dem nicht. Insofern, als sie (reines) Eigeninteresse als Motivation unterstellen und damit nur den instrumentellen Wert der Wahlbeteiligung berücksichtigen, liegen sie mit ihrer Kritik richtig. Andererseits ist dies wiederum ein Beispiel dafür, in welche Probleme man gerät, wenn man zwischen den Annahmen der Rationalität und des Eigeninteresses nicht unterscheidet.

28 Zur Geschichte des Allais-Paradox vgl. Schoemaker (1982: 541ff.). 
nalität zu sprechen. Dies wird deutlich, wenn wir berücksichtigen, dass diese Inkonsistenzen verschwinden, sobald sich die Betroffenen der inhaltlichen Übereinstimmung der beiden Darstellungen bewusst werden.

Ein stärkeres Argument gegen das Konzept der schwachen Rationalität mag von jenen Experimenten abgeleitet werden, in denen eine Präferenzumkehr beobachtet werden kann. Hier wird scheinbar die oben angesprochene Transitivitätsannahme verletzt (vgl. Lichtenstein/Slovic 1971; Pommerehne et al. 1982; Guala 2000; sowie die Übersicht bei Tversky/Thaler 1990). Dass Präferenzen „konsistente und vorhersagbare Intransitivitäten“ aufweisen können, wurde bereits von Tversky (1969: 31) aufgezeigt. Was dabei tatsächlich verletzt wird, ist die Konsistenz über die Zeit hinweg. Dies kann wiederum ein Problem unterschiedlicher Darstellungen (Frames) sein. Wenn aber, wie Tversky (1969) ausführt, dieses Verhalten mit der ,Regret-Theorie` vereinbar ist, wird offensichtlich, dass wir es hier wieder mit einem Problem der traditionellen Rationalitätsannahme und nicht mit einem Problem des schwachen Rationalitätsprinzips zu tun haben: Seine Argumentation basiert auf einem allgemeinen Wahlhandlungsmodell.

Gegen all dies mag eingewandt werden, dass das Verhalten der Menschen in der Realität weniger durch rationale (oder begrenzt rationale) Entscheidungen charakterisiert wird als vielmehr durch Befolgen rechtlicher oder sozialer Normen. Dieses Modell menschlichen Verhaltens wurde z.B. durch Dahrendorf (1958) in seinem „Homo Soziologicus" vorgestellt. Man mag dies als ein Modell in der nicht-individualistischen Tradition der Soziologie interpretieren, die auf Durkheim (1961/1895) zurückgeht. Da die Menschen nur selten wirklich überlegte Entscheidungen treffen und meist intuitiv entscheiden, wird dieses Modell dem ökonomischen Ansatz gerne als ein ,realistischeres $^{\text {' }}$ Modell entgegengesetzt.

Solange man Regeln nicht absolut strikt, d.h. völlig unabhängig von der Situation sowie von seinen Ergebnissen befolgt, ist das Handeln nach Regeln jedoch vollständig vereinbar mit dem schwachen Rationalitätsprinzip. Im Modell von Dahrendorf (1958) reagieren die Individuen auf Sanktionen, d.h. auf Anreize, genau wie im ökonomischen Ansatz. Außerdem weist König (1961: 36f.) darauf hin, dass es möglich ist, das berühmteste Werk von Durkheim, seinen „Selbstmord“ (1995/1897), im Kontext einer individualistischen Sozialtheorie zu re-interpretieren. Durkheims hauptsächliche Intention war es zu zeigen, dass die sozialen Fakten, d.h. die gesellschaftlichen Wirkungen individuellen Handelns, nicht direkt aus den individuellen Intentionen ableitbar sind, sondern sich oft als nicht-intendierte Konsequenzen dieses Handeln ergeben, und dass sie wesentlich durch gesellschaftliche Bedingungen beeinflusst werden. Insofern befindet sich dieser Ansatz in völliger Übereinstimmung mit Adam Smith (1974/1776) bzw. mit der ökonomischen Tradition. Unabhängig davon, dass es soziologische Theorien gibt, die nur sehr schwer - oder möglicherweise auch gar nicht individuell rekonstruierbar sind, bietet die soziologische Rollentheorie keinen Einwand gegen das schwache Rationalitätsprinzip.

Abschließend ist zu diskutieren, ob das sich neu entwickelnde Feld der ,Neuroökonomie' Zweifel an der Gültigkeit des schwachen Rationalitätsprinzips rechtfertigt. ${ }^{29}$

29 Zu neueren Übersichten über die Neuroökonomie vgl. z.B. Fehr/Rangel 2011 sowie die Beiträge im Journal of Economic Methodology, Vol. 17/No. 2, (2010). 
Zum einen kann dies offensichtlich nicht der Fall sein, da dieses Prinzip keinen empirischen Gehalt hat (und damit durch Ergebnisse empirischer Forschung auch nicht widerlegt werden kann). Andererseits könnten einige Ergebnisse aus diesem neuen Gebiet sogar starke Versionen des Rationalitätsprinzips rechtfertigen. Durch Analyse der Gehirnaktivität zeigen z.B. de Quervain et al. (2004), dass bei Bestrafungshandlungen, die für den Bestrafenden mit Aufwand verbunden sind, die gleichen Gehirnzellen aktiviert werden wie bei anderen Aktivitäten, die (im traditionellen Sinn) dem Akteur Befriedigung verschaffen, wie z.B. der Konsum von Gütern. Damit ist es möglich, solche psychologischen Effekte in nicht-tautologischer (und damit auch empirisch überprüfbarer) Weise in das ökonomische Verhaltensmodell einzubauen. In gewisser Weise wird damit sogar das traditionelle allgemeine Modell des homo oeconomicus rehabilitiert: Er versucht, ein möglichst hohes $\mathrm{Maß}$ an Befriedigung zu erlangen, wobei solche Befriedigungen auch mit Handlungen verbunden sein können, die der engen, auf rein monetäre Aspekte abhebenden Interpretation des Eigeninteresses widersprechen. Der hier betrachtete Mensch steigert seinen Nutzen auch durch Handlungen, die kostspielig sind, ohne ihm zusätzliche Konsumchancen einzuräumen. Hier ergibt sich nirgends ein Widerspruch mit dem schwachen Rationalitätsprinzip.

Möglicherweise könnte jedoch, was bisher ein heuristisches Prinzip war, durch neuroökonomische Untersuchungen in eine überprüfbare Hypothese überführt werden. Insofern mag die Neuroökonomie zu einer Mikrofundierung der Mikrofundierung der Wirtschaftswissenschaften (sowie anderer Theorien der Gesellschaft) führen. Ob dies gelingt, ist fraglich, und welche Konsequenzen dies für die Erklärung von Makrophänomenen sowie insbesondere für deren Anwendung in der praktischen Wirtschaftspolitik haben könnte, ist derzeit noch nicht absehbar.

\section{Abschließende Bemerkungen}

Das schwache Rationalitätsprinzip ist ein heuristisches Prinzip, welches Anleitung für jede, verstehende“ Sozialwissenschaft im Sinne von Max Weber gibt. Es hat keinen empirischen Gehalt und kann daher durch empirische Argumente auch nicht verworfen werden, weder durch die Ergebnisse von Experimenten, noch durch jene von Feldstudien. Es könnte jedoch aus praktischen Gründen obsolet werden, wenn man z.B. mit Hilfe eines anderen heuristischen Prinzips zu ,besseren' Einsichten in gesellschaftliche Beziehungen und damit zu einer fruchtbareren Theorie der Gesellschaft gelangen würde. Ob dies der Fall ist oder nicht, soll hier nicht diskutiert werden, auch wenn es schwer ist, sich eine Alternative vorzustellen, welche dem ökonomischen Ansatz generell überlegen wäre. Dies schließt nicht aus, dass andere Ansätze bei der Erklärung bestimmter Phänomene ,besser' abschneiden.

Empirische Überprüfungen sind prinzipiell immer (und oft auf sehr einfache Art und Weise) möglich, sobald zusätzliche Annahmen über die Präferenzen und/oder die verfügbare Information getroffen werden, wenn zum schwachen Rationalitätsprinzip zusätzliche Annahmen hinzukommen. Dies gilt, z.B. für verschiedene Tests auf ,rationale Erwartungen' (vgl. hierzu z.B. Kirchgässner 1993). Aber dann werden verbundene Hypothesen getestet, und wenn ein Ansatz verworfen wird, ist es in aller Regel sehr schwierig oder gar unmöglich zu entscheiden, woran das liegt, d.h. welche der verschiedenen Annahmen dafür verantwortlich ist. 
Ein Vorteil der Anwendung des (schwachen) Rationalitätsprinzips ist auch, dass es zwei miteinander nur schwer verträgliche Perspektiven menschlichen Handelns vereinbar macht: die Annahme des freien Willens und die Vorhersagbarkeit menschlichen Verhaltens. Um das Verhalten eines Individuums prognostizieren zu können, spielt es keine Rolle, ob dieses einem deterministischen Gesetz folgt oder ob es in einer bestimmten Art und Weise rational entscheidet (vgl. hierzu Lagueux 2004: 32). Ex post können wir selbstverständlich versuchen, jedes Verhalten zu verstehen. Dies hilft uns aber nicht unbedingt, zukünftiges Verhalten zu prognostizieren. Hierzu müssen wir Modelle konstruieren, welche die typische Situation, in der sich die Individuen befinden, repräsentieren: die „Situationslogik“, um einen Begriff von Popper (1962: 120) zu verwenden. Da individuelle Entscheidungen jedoch von vielen Faktoren abhängen, die in unseren Mikromodellen nicht explizit berücksichtigt werden können, eignet sich das Rationalitätsprinzip ex ante eher für die Vorhersage aggregierter Ergebnisse als zur Prognose individuellen Verhaltens, zumindest soweit sich die Abweichungen der individuellen Verhaltensweisen von der Lösung, die vom Modell unterstellt wird, (zumindest weitgehend) ausgleichen (vgl. hierzu auch Zintl 1989).

Schließlich ist das schwache Rationalitätsprinzip auch mit der Idee hierarchisch geordneter Präferenzordnungen vereinbar. Probleme wie die ,Willensschwäche' und die Beobachtungen, dass Individuen gelegentlich, um bestimmte Ziele zu erreichen, ihren zukünftigen Handlungsraum freiwillig einschränken, indem sie Selbstbindungen eingehen, sind mit dem traditionellen ökonomischen Ansatz kaum vereinbar. ${ }^{30} \mathrm{Um}$ diese Phänomene zu erfassen, muss man annehmen, dass die Individuen zwei Arten von Präferenzen haben, die geordnet sind, bzw. dass sie Präferenzen auf zwei verschiedenen Ebenen haben (vgl. hierzu auch Schelling 1978, 1980; Sen 1977; Elster 1986; sowie Thaler/Shefrin 1981). Auf der unteren Ebene befinden sich die hier und heute gegebenen Präferenzen, entsprechend denen sich das Individuum in einer bestimmten Situation entscheidet. Auf der übergeordneten Ebene liegen jene Präferenzen, gemäß denen das Individuum sich gerne handeln sähe. Beide Präferenzordnungen können und werden in aller Regel in sich konsistent sein, aber zwischen den beiden Ebenen können sich Widersprüche ergeben. ${ }^{31}$ Solange keine konkreten Handlungen notwendig sind, kann das Individuum entsprechend seiner übergeordneten Präferenzen ,planen' und dabei die später in den konkreten Situationen auftretenden Restriktionen so zu gestalten versuchen, dass die tatsächlichen Handlungen sich dann so weit als möglich an den übergeordneten Präferenzen orientieren.

Die Vorstellung zweier unterschiedlicher und sich zumindest zum Teil widersprechender Präferenzordnungen mag auf den ersten Blick befremdlich erscheinen, und sie ist zumindest für denjenigen, der von der Ökonomik her kommt und gewohnt ist, mit eindeutigen (und festen) Präferenzen der Individuen zu argumentieren, eher ungewöhnlich. Dies gilt jedoch nicht für die philosophische Tradition: Hier kann diese Vorstellung zumindest bis auf Aristoteles zurückverfolgt werden, der in seiner „Ni-

30 Eine ganze Reihe von Beispielen für Selbstbindungen findet sich bei Schelling (1984). Viele dieser Beispiele stammen aus dem täglichen Leben.

31 Besonders deutlich sind diese Widersprüche bei Süchtigen, die gegen ihre Sucht anzugehen versuchen. 
komachischen Ethik“ (1138b) von zwei verschiedenen Seelenteilen, einem vernünftigen und einem unvernünftigen spricht, deren Verhältnis dem zwischen Herrscher und Untertan entsprechen solle. Und wie Frankfurt (1971) ausführt, sind die Existenz übergeordneter Präferenzen und die Möglichkeit, mit ihrer Hilfe die untergeordneten Präferenzen zu beurteilen, sogar eine Voraussetzung dafür, einen Menschen als ,Person' mit freiem Willen zu sehen, denn hierfür ist notwendig, dass man sich dieses seines Willens bewusst ist. Genau das geschieht aber, wenn Individuen versuchen, sich selbst zu binden. Die Idee und sogar die Terminologie von Aristoteles wurden z.B. durch Thaler/Shefrin (1981) in ihrem ökonomischen Modell der Selbstkontrolle aufgegriffen, wenn sie vom „Planer“ und vom „Ausführenden“ („Doer“) sprechen, die unterschiedliche Präferenzordnungen haben.

Wie seine Anwendung in den Wirtschaftswissenschaften gezeigt hat, ist das schwache Rationalitätsprinzip eine sehr hilfreiche methodische Richtschnur für gesellschaftswissenschaftliche Analysen, die auch in andern Sozialwissenschaften Anwendung finden kann und auch findet. Welche zusätzlichen Annahmen dabei erforderlich sind, hängt vom Bereich ab, in dem dieser Ansatz angewendet wird. Inwieweit sich diese Anwendungen als fruchtbar erweisen, hängt weitgehend davon ab, inwieweit alternative, fruchtbare Ansätze vorhanden sind, insbesondere solche, die nicht auf dem Konzept einer verstehenden Sozialwissenschaft im Sinne vom Max Weber fußen. Solange man aber in diesem Rahmen bleibt, kann man auf das schwache Rationalitätsprinzip nicht verzichten.

\section{Literaturverzeichnis}

Abel, B. (1983): Grundlagen der Erklärung menschlichen Handelns, Tübingen: Mohr Siebeck.

Albert, H. (1964): Der Mythos der totalen Vernunft: Dialektische Ansprüche im Lichte undialektischer Kritik, Kölner Zeitschrift für Soziologie und Sozialpsychologie, Vol. 16/No. 2, 225-256.

Allais, $M$ (1953): Le comportement de l'homme rationnel devant risque: Critique des postulats et axiomes de l'école Américaine, Econometrica, Vol. 21/No. 4, 503-546; ausführlichere englische Version: The Foundations of a Positive Theory of Choice Involving Risk and a Criticism of the Postulate and Axioms of the American School, in: Allais M./Hagen O. (1979) (Eds.): Expected Utility Hypotheses and the Allais Paradox, Dordrecht: Reidel, 27-145.

Andreoni J. (1988): Privately Produced Public Goods in a Large Economy: The Limits of Altruism, in: Journal of Public Economics, Vol. 35/No. 1, 57-73.

Andreoni J. (1989): Giving with Impure Altruism: Applications to Charity and Ricardian Equivalence, in: Journal of Political Economy, Vol. 97/No. 6, 1447-1458.

Andreoni J. (1990): Impure Altruism and Donations to Public Goods: A Theory of Warm Glow Giving, in: Economic Journal, Vol. 100/No. 401, 464-477.

Angehrn E. (1983): Handlungserklärung und Rationalität, Zur Methodologie Max Webers, in: Zeitschrift für philosophische Forschung, Vol. 37/No. 3, 341-362.

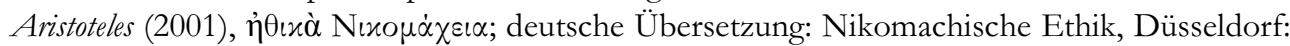
Artemis und Winkler.

Arrow, K. J. (1986): Rationality of Self and Others in an Economic System, in: Journal of Business, Vol. 59/No. 4, 385-399. 
Becker, G. S. (1962): Irrational Behavior and Economic Theory, in: Journal of Political Economy, Vol. 70/No. 1, 1-13.

Binmore, K. (2009): Rational Decisions, Princeton, Oxford: Princeton University Press.

Bühler, A. (1987): Die Einheit der wissenschaftlichen Methode und Maximen des Verstehens, in: Zeitschrift für philosophische Forschung, Vol. 41/No. 4, 633-644.

Bunge, M. (1959): Causality: The Place of the Causal Principle in Modern Science, Cambridge (Mass.): Harvard University Press.

Conlisk, J. (1996): Why Bounded Rationality?, in: Journal of Economic Literature, Vol. 34/No. 2, 669-700.

Dahrendorf, R. (1958): Homo Sociologicus: Versuch zur Geschichte, Bedeutung und Kritik der Kategorie der sozialen Rolle, Kölner Zeitschrift für Soziologie und Sozialpsychologie, Vol. 10/No. 2, 178-208; abgedruckt in: Dahrendorf, R. (1967): Pfade aus Utopia: Arbeiten zur Theorie und Methode der Soziologie, München: Piper, 194-211.

Durkheim, E. (1961/1895): Les régles de la méthode sociologique, Paris: Les Presses Universitaires de France; deutsche Übersetzung: Die Regeln der soziologischen Methode, Neuwied: Luchterhand.

Durkheim, E. (1995/1897): Le suicide, Etude de sociologie, Paris: Les Presses Universitaires de France; deutsche Übersetzung: Der Selbstmord, Frankfurt a.M.: Suhrkamp.

Elster, J. (1979): Ulysses and the Sirens, Studies in Rationality and Irrationality, Cambridge: Cambridge University Press.

Elster, J. (1986): The Multiple Self, Cambridge: Cambridge University Press.

Evans, C. L. (1994): The Post-War U.S. Phillips Curve: A Comment, in: Carnegie-Rochester Conference Series on Public Policy, Vol. 41, 221-230.

Fehr, E./Gächter, S. (1998): Reciprocity and Economics: The Economic Implications of Homo Reciprocans, in: European Economic Review, Vol. 42/No. 3-5, 845-859.

Fehr, E./Gächter, S. (2000): Fairness and Retaliation: The Economics of Reciprocity, in: Journal of Economic Perspectives, Vol. 14/No. 3, 159-181.

Fehr, E./Rangel, A. (2011): Neuroeconomic Foundations of Economic Choice: Recent Advances, in: Journal of Economic Perspectives, Vol. 25/No. 4, 3-30.

Fehr, E./Schmidt, K. M. (1999): A Theory of Fairness, Competition, and Cooperation, in: Quarterly Journal of Economics, Vol. 114/No. 3, 817-868.

Fehr, E./Tyran, J.-R. (2001): Does Money Illusion Matter?, in: American Economic Review, Vol. 91/No. 6, 1239-1262.

Frankfurt, H. G. (1971): Freedom of the Will and the Concept of a Person, in: Journal of Philosophy, Vol. 68/No. 1, 5-20.

Frings, A. (2007): Rationales Handeln und historische Erklärung, Journal for General Philosophy of Science, Vol. 38/No. 1, 31-56.

Green, D. P./Shapiro, I. (1994): Pathologies of Rational Choice: A Critique of Applications in Political Science, New Haven: Yale University Press.

Guala, F. (2000): Artefacts in Experimental Economics: Preference Reversals and the BeckerDegroot-Marschak Mechanism, in: Economics and Philosophy, Vol. 16/No. 1, 47-75.

Habermas, J. (1964): Gegen einen positivistisch halbierten Rationalismus: Erwiderung eines Pamphlets, in: Kölner Zeitschrift für Soziologie und Sozialpsychologie, Vol. 16/No. 4, 636-659.

Hands, D. W. (1985): Karl Popper and Economic Methodology, in: Economics and Philosophy, Vol. 1/No. 1, 63-99.

Harstad, R. M./Selten, R. (2013): Bounded Rationality Models: Tasks to Become Intellectually Competitive, in: Journal of Economic Literature, Vol. 51/No. 2, 496-511. 
Hausman, D. M./McPherson, M. S. (1996), Economic Analysis and Moral Philosophy, Cambridge (U.K.): Cambridge University Press.

Hayek, F. A. (1952): The Counter Revolution of Science: Studies in the Abuse of Reason, Glencoe: The Free Press.

Häring, N. (2001), Der Homo Oeconomicus ist tot, Financial Times Deutschland vom 14. März 2001. Link: http://ockenfels.uni-koeln.de/fileadmin/wiso_fak/stawi-ockenfels/ pdf/Presse/Der_Homo_oeconomicus_ist_tot.pdf (zuletzt abgerufen am 26.06.2012).

Heiner, R. A. (1983): The Origin of Predictable Behavior, in: American Economic Review, Vol. 73/No. 4, 560-595.

Heiner, R. A. (1990): Rule Governed Behavior in Evolution and Human Society, in: Constitutional Political Economy, Vol. 1/No. 1, 19-46.

Hicks, J. R. (1939): Value and Capital, An Inquiry into Some Fundamental Principles of Economic Theory, Oxford: Oxford University Press, second edition 1946.

Hume, D. (1978/1739): A Treatise of Human Nature, Oxford: Clarendon Press.

King, R. G./Watson, M. W. (1994): The Post-War U.S. Phillips Curve, in: Carnegie-Rochester Conference Series on Public Policy, Vol. 41, 157-219.

Kirchgässner, G. (1985): Rationales Verhalten und vernünftiges Handeln, ein Widerspruch?, in: Milde, H./Monissen, H. G. (Eds.): Rationale Wirtschaftspolitik in komplexen Gesellschaften, Stuttgart: Kohlhammer, 29-41.

Kirchgässner, G. (1992): Towards a Theory of Low-Cost Decisions, in: European Journal of Political Economy, Vol. 8/No. 2, 305-320.

Kirchgässner, G. (1993): Testing Weak Rationality of Forecasts with Different Time Horizons, in: Journal of Forecasting, Vol. 12/No. 7, 541-558.

Kirchgässner, G. (2002): On the Role of Heroes in Political and Economic Processes, in: Kyklos, Vol. 55/No. 2, 179-196.

Kirchgässner, G. (2008): Homo Oeconomicus, Das ökonomische Modell individuellen Verhaltens und seine Anwendung in den Wirtschafts- und Sozialwissenschaften, Tübingen: Mohr Siebeck, 3. Auflage.

Kirchgässner, G. (2010): On Minimal Morals, in: European Journal of Political Economy, Vol. 26/No. 3, 330-339.

Kirchgässner, G. (2013): The Weak Rationality Principle in Economics, in: Schweizerische Zeitschrift für Volkswirtschaft und Statistik, Vol. 149/No. 1, 1-26.

Kliemt, H. (1984): Nicht-explanative Funktionen eines 'Homo oeconomicus' und Beschränkungen seiner explanativen Rolle, in: Holler, M.J. (Ed.): Homo oeconomicus II, München: Leudemann, 7-49.

König, R. (1961): Einleitung, zu: E. Durkheim, Die Regeln der soziologischen Methode, Neuwied: Luchterhand (1961), 19-82.

Lagueux, M. (2004): The Forgotten Role of the Rationality Principle in Economics, in: Journal of Economic Methodology, Vol. 11/No. 1, 31-51.

Lagueux, M. (2010): Rationality and Explanation in Economics, London, New York: Routledge.

Latsis, S. J. (1983): The Role and Status of the Rationality Principle in the Social Sciences, in: Cohen, R. S./Wartofsky, M. W. (Eds.): Epistemology, Methodology and the Social Sciences, Dordrecht, Boston: Reidel, 123-151.

Lichtenstein, S./Slovic, P. (1971): Reversals of Preference Between Bids and Choices in Gambling Decisions, in: Journal of Experimental Psychology, Vol. 89/No. 1, 46-55.

Lucas, R. E. (1976): Econometric Policy Evaluation, A Critique, in: Carnegie-Rochester Conference Series on Public Policy, Vol. 1, 19-46. 
Machlup, F. (1960): Der Wettstreit zwischen Mikro- und Makrotheorien der Nationalökonomie, Tübingen: Mohr (Siebeck).

Menger, C. (1883): Untersuchungen über die Methode der Sozialwissenschaften und der politischen Ökonomie insbesondere, Leipzig: Duncker und Humblot.

Muth, J. E. (1961): Rational Expectations and the Theory of Price Movements, Econometrica, Vol. 29/No. 3, 315-335.

Neumann, J./Morgenstern, O. (1944): Theory of Games and Economic Behavior, Princeton: Princeton University Press.

North, D. C. (1981): Structure and Change in Economic History, New York: Norton.

Nozick, R. (1993): The Nature of Rationality, Princeton: Princeton University Press.

Päbler, K. (1986): Qualitätsmerkmale wissenschaftlicher Theorien: Zur Logik und Ökonomik der Forschung, Tübingen: Mohr Siebeck.

Patzak, B. M. (1984): Rationalmodell und analytische Handlungstheorie, in: Holler, M.J. (Hrsg.): Homo oeconomicus III, München: Leudemann, 7-42.

Phillips, A. W. (1958): The Relation Between Unemployment and the Rate of Change of Money Wage Rates in the United Kingdom, 1861-1957, in: Economica, Vol. 25/No. 100, 283-299.

Pommerehne, W.W./Schneider, F./Zweifel P. (1982): Economic Theory of Choice and the Preference Reversal Phenomenon, A Reexamination, in: American Economic Review, Vol. 72/No. 3, 569-574.

Popper, K. R. (1962): Die Logik der Sozialwissenschaften, in: Kölner Zeitschrift für Soziologie und Sozialpsychologie, Vol. 14/No. 2, 233-248; abgedruckt in und zitiert nach: Adorno, Th. W. et al. (1969): Der Positivismusstreit in der deutschen Soziologie, Frankfurt a.M.: Luchterhand, 103-123.

Popper, K. R. (1967): La rationalité et le statut du principe de rationalité, in : Classen, E. M. (Ed.): Les fondements philosophiques des systèmes économiques: Textes de Jaques Rueff et essais rédigés en son honneur, Paris: Payot, 142-150; englische Übersetzung: The Rationality Principle, in: Miller, D. (Ed.) (1985): Popper Selections, Princeton: Princeton University Press, 257-365; abgedruckt in: Caldwell, B. J. (Ed.) (1993): The Philosophy and Methodology of Economics, Volume III, Aldershot: Edward Elgar, 311.

Popper, K. R. (1972): Objective Knowledge, Clarendon Press: Oxford 1972; deutsche Übersetzung: Objektive Erkenntnis, Ein evolutionärer Entwurf, Hamburg: Hoffmann und Campe.

Quervain, D. J.-F./Fischbacher, U./Treyer, V./Schellhammer, M./Schnyder, U./Buck, A./Fehr, E. (2004): The Neural Basis of Altruistic Punishment, in: Science, Vol. 305/No. 5688, 1254-1258.

Ramser, H. (1987): Beschäftigung und Konjunktur, Berlin: Springer.

Riker, W. H./Ordeshook, P. C. (1968): A Theory of the Calculus of Voting, in: American Political Science Review, Vol. 62/No. 1, 25-42.

Rubinstein, A. (1998): Modeling Bounded Rationality, Cambridge (Mass.): MIT Press.

Samuelson P. A./Solow, R. M. (1960): Analytical Aspects of Anti-Inflation Policy, in: American Economic Review, Papers and Proceedings, Vol. 50/No. 2, 177-194.

Santomero, A. M./Seater, J. J. (1978): The Inflation-Unemployment Trade-off: A Critique of the Literature, in: Journal of Economic Literature, Vol. 16/No. 2, 499-544.

Schelling, T. C. (1978): Egonomics, or the Art of Self-Management, in: American Economic Review, Papers and Proceedings, Vol. 68/No. 2, 290-294. 
Schelling, T. C. (1980): The Intimate Contest for Self-Command, in: Public Interest, Vol. 60, 94-118.

Schelling, T. C. (1984): Self-Command in Practice, in Policy, and in a Theory of Rational Choice, in: American Economic Review, Papers and Proceedings, Vol. 74/No. 2, 1-11.

Schlicht, E. (1977): Grundlagen der ökonomischen Analyse, Hamburg: Rowohlt.

Schmid, M. (1979a): Handlungsrationalität, Kritik einer dogmatischen Handlungswissenschaft, München: Wilhelm Fink.

Schmid, M. (1979b): Rationalitätsprinzip und Handlungserklärung, in: Lenk, H. (Hrsg.): Handlungstheorien-interdisziplinär, Vol. 2.1, München: Wilhelm Fink, 491-533.

Schoemaker, P. J. H. (1982): The Expected Utility Modell: Its Variants, Purposes, Evidence and Limitations, in: Journal of Economic Literature, Vol. 20/No. 2, 529-563.

Schumpeter, J. A. (1965): Geschichte der ökonomischen Analyse, Göttingen: Vandenhoeck und Ruprecht.

Schurz, G. (Hrsg.) (1988): Erklären und Verstehen in der Wissenschaft, München: Oldenbourg.

Searle, J. R. (2001): The Classical Model of Rationality and Its Weaknesses, in: Searle, J. R. (Ed.): Rationality in Action, Cambridge (Mass.): MIT Press, 1-32.

Selten, R. (1990): Bounded Rationality, in: Zeitschrift für die gesamte Staatswissenschaft (JITE), Vol. 146/No. 4, 649-658.

Selten, R. (2001): What is Bounded Rationality?, in: Gigerenzer, G./Selten, R. (Eds.): Bounded Rationality: The Adaptive Toolbox, Cambridge (Mass): MIT Press, 13-36.

Sen, A. K. (1977): Rational Fools: A Critique of the Behavioural Foundations of Economic Theory, in: Philosophy and Public Affairs, Vol. 6/No. 4, 317-344.

Sen, A. K. (1985): Goals, Commitment, and Identity, in: Journal of Law, Economics, and Organization, Vol. 1/No. 2, 341-355.

Sen, A. K. (1987): Rational Behaviour, in: The New Palgrave: A Dictionary of Economics, Vol. 4, London: Macmillan, 68-76.

Sen, A. K. (1994): The Formulation of Rational Choice, in: American Economic Review, Papers and Proceedings, Vol. 84/No. 2, 385-390.

Sen, A. K. (2005): Why Exactly is Commitment Important for Rationality, in: Economics and Philosophy, Vol. 21/No. 1, 5-13.

Sen, A. K./Williams, B. (1982): Introduction, in: Sen, A. K./Williams, B. (Eds.): Utilitarianism and Beyond, Cambridge (England) et al.: Cambridge University Press, 1-21.

Simon, H. A. (1955): A Behavioral Model of Rational Choice, in: Quarterly Journal of Economics, Vol. 69/No. 1, 99-118.

Simon, H. A. (1978): Rationality as Process and as Product of Thought, in: American Economic Review, Papers and Proceedings, Vol. 68/No. 2, 1-16.

Simon, H. A. (1979): Rational Decision Making in Business Organizations, in: American Economic Review, Vol. 69/No. 4, 493-513.

Smith, A. (1974/1776): An Inquiry into the Nature and Causes of the Wealth of Nations, London; deutsche Übersetzung: Der Wohlstand der Nationen, Eine Untersuchung seiner Natur und seiner Ursachen, München: C.H. Beck.

Stegmüller, W. (1960): Das Problem der Kausalität, in: Topitsch, E. (Hrsg.): Probleme der Wissenschaftstheorie, Festschrift für Viktor Kraft, Wien: Springer, 171-190.

Stegmüller, W. (1969): Probleme und Resultate der Wissenschaftstheorie und analytischen Philosophie, Band I, Wissenschaftliche Erklärung und Begründung, Berlin: Springer

Stocké, V. (2002): Framing und Rationalität: Die Bedeutung der Informationsdarstellung für das Entscheidungsverhalten, München: Oldenbourg. 
Sudgen, R. (1985): Why Be Consistent? A Critical Analysis of Consistency Requirements in Choice Theory, in: Economica, Vol. 52/No. 206, 167-183.

Thaler, R. H. (1992): The Winner's Curse, Paradoxes and Anomalies of Economic Life, New York: The Free Press.

Thaler, R. H./Shefrin H. M. (1981): An Economic Theory of Self-Control, in: Journal of Political Economy, Vol. 89/No. 2, 392-406.

Tietzel, M. (1981): Die Rationalitätsannahme in den Wirtschaftswissenschaften, oder: Der homo oeconomicus und seine Verwandten, in: Jahrbuch für Sozialwissenschaft, Vol. 32/No. 2, 115-138.

Tversky, A. (1969): Intransitivity of Preferences, in: Psychological Review, Vol. 76/No. 1, 31-48.

Tversky, A./Kahneman, D. (1981): The Framing of Decisions and the Psychology of Choice, in: Science, Vol. 211/No. 4481, 453-458.

Tversky, A./Kabneman, D. (1987): Rational Choice and the Framing of Decisions, in: Hogarth, R. M./Reder, M. W. (Eds.): Rational Choice: The Contrast between Economics and Psychology, Chicago: Chicago University Press, 67-94.

Tversky, A./Thaler, R. H. (1990): Anomalies: Preference Reversals, in: Journal of Economic Perspectives, Vol. 4/No. 2, 201-211.

Vanberg, V. (1975): Die zwei Soziologien, Tübingen: Mohr Siebeck.

Vanberg, V. (2004): The Rationality Postulate in Economics: Its Ambiguity, Its Deficiency and Its Evolutionary Alternative, in: Journal of Economic Methodology, Vol. 11/No. 1, $1-29$.

Watkins, J. W. N. (1957): Between Analytical and Empirical, in: Philosophy, Vol. 32/No. 121, 112-131.

Watkins, J. W. N. (1958): Confirmable and Influential Metaphysics, in: Mind, Vol. 67/No. 267, 344-365.

Watkins, J. W. N. (1975): Metaphysics and the Advancement of Science, in: British Journal for the Philosophy of Science, Vol. 26/No. 2, 91-121.

Watkins, J. W. N. (1978): Freiheit und Entscheidung, Tübingen: Mohr Siebeck.

Weber, M. (1922): Wirtschaft und Gesellschaft, Tübingen: Mohr Siebeck.

Zintl, R. (1989): Der Homo Oeconomicus: Ausnahmeerscheinung in jeder Situation oder Jedermann in Ausnahmesituationen, in: Analyse und Kritik, Vol. 11/No. 1, 52-69. 